Advance Access publication 2016 August 4

GJI Geomagnetism, rock magnetism and palaeomagnetism

\title{
Dynamos driven by helical waves: scaling laws for numerical dynamos and for the planets
}

\author{
P. A. Davidson \\ Department of Engineering, University of Cambridge,United Kingdom.E-mail: pad3@eng.cam.ac.uk
}

Accepted 2016 August 3. Received 2016 August 3; in original form 2016 June 6

\begin{abstract}
S UMMAR Y
We derive scaling relationships for planetary dynamos based on a balance between energy production and Joule dissipation, and between the curl of the buoyancy and Coriolis forces. These scaling relationships are deduced for the particular case of dynamos driven by helical waves, but are shown to have a much broader applicability. They are consistent with the evidence of the numerical dynamos, yielding predictions consistent with published empirical scaling laws and also with the observed transition from dipolar to multipolar dynamos. A direct comparison with the observational evidence for the planets is hampered by the fact that we do not know what sets the smallest scale of the motion in the planets. Nevertheless, we use our scaling relationships to show that the traditional assumption that the Elsasser number is of order unity is inconsistent with the observation that the gas-giant dynamos are dipolar dynamos, as is the more recent suggestion that the strength of the dipole is independent of rotation rate and controlled by the buoyancy flux alone. On the other hand, we show that the observational data is consistent with the hypothesis that a dipolar dynamo saturates at the lowest permissible magnetic energy compatible with a given buoyancy flux.
\end{abstract}

Key words: Electromagnetic theory; Dynamo: theories and simulations; Geomagnetic induction.

\section{INTRODUCTION}

One of the notable successes in planetary dynamo theory over the last two decades has been the ability of the numerical simulations to reproduce plausible looking magnetic fields, that is dipoles aligned with the planetary rotation axis (Glatzmaier \& Roberts 1995). Some of the geodynamo simulations even manage to reproduce more intricate details, such as the observed westward drift (Aubert et al. 2013). This is all the more surprising since the numerical dynamos operate in a parameter regime quite unlike the planets, being too viscous by a factor of $10^{9}$, as measured by the Ekman number, and underpowered by a factor of $10^{3}$, relative to the onset of convection. So, given that many of their features must be distinctly unlike a planet, perhaps the most pertinent questions to ask of the current geodynamo simulations, are: (i) what are the simulations getting right that allows them to produce such plausible looking fields? and (ii) what are the key differences in behaviour between the simulations and the planets?

Given the success of the simulations, many authors have tried to establish dynamo scaling laws based on the results of the numerical dynamos. These laws attempt to relate the rate of working of the buoyancy forces, which powers such dynamos, to the strength of the induced dipole field and the intensity of the convective motion. Typically the scaling laws are established empirically using a suite of numerical dynamos which span a range of parameter values, though they are usually guided by simple proposed force balances or kinematic constraints. (See, for example, Christensen \& Aubert 2006; Christensen 2010; Schrinner et al. 2012; Yadav et al. 2012; Stelzer \& Jackson 2013; Davidson 2014; Oruba \& Dormy 2014.) Many such scalings have now been proposed, and the hope of some authors is that this may allow the results of the numerical dynamos to be extrapolated to the planets. However, given that the numerical and planetary dynamos inhabit such different dynamical regimes, any simple interpolation between the two must be regarded with some caution.

Table 1 shows the properties of the planets, and in particular their magnetic field strengths. The observed magnetic fields of Mercury, the Earth, Jupiter and Saturn are all rather similar, being strongly dipolar with the magnetic axis more or less aligned with the rotation axis. On the other hand, the fields of the ice giants are not at all dipolar, which probably reflects a very different dynamo regime operating within their cores. In Table 1 the magnitude of the averaged axial magnetic field in the conducting core, $\bar{B}_{z}$, is obtained from the observed dipole moment, $\mathbf{m}$, using (1), a well-known expression relating the dipole moment associated with currents, $\mathbf{J}$, confined to a sphere of volume $V_{C}$, to the integral of the magnetic field over $V_{C}$ :

$\mathbf{m}=\frac{1}{2} \int_{V_{C}} \mathbf{x} \times \mathbf{J} \mathrm{d} V=\frac{3}{2 \mu} \int_{V_{C}} \mathbf{B} \mathrm{d} V$. 
Table 1. Properties of the planets. Note the relatively uniform value of the normalised magnetic field, $\bar{B}_{z} / \Omega R_{C} \sqrt{\rho \mu}$, but the large variation in the Elsasser number, $\Lambda$. We use the estimates of $\lambda \sim 0.7 \mathrm{~m}^{2} \mathrm{~s}^{-1}, \rho \sim 10^{4} \mathrm{~kg} \mathrm{~m}^{-3}$ for the terrestrial planets, $\lambda \sim 3 \mathrm{~m}^{2} \mathrm{~s}^{-1}, \rho \sim 10^{3} \mathrm{~kg} \mathrm{~m}^{-3}$ for the gas giants, and $\lambda \sim 300 \mathrm{~m}^{2} \mathrm{~s}^{-1}, \rho \sim 4 \times 10^{3} \mathrm{~kg} \mathrm{~m}^{-3}$ for the ice giants. The values of $\mathbf{m}$ are from Davidson (2013) and references therein.

\begin{tabular}{|c|c|c|c|c|c|c|c|c|}
\hline Planet & $\begin{array}{l}\text { Rotation } \\
\text { period }(d)\end{array}$ & $\begin{array}{l}\text { Radius of planet } \\
\qquad\left(10^{3} \mathrm{~km}\right)\end{array}$ & $\begin{array}{l}\text { Radius of core, } \\
R_{C}\left(10^{3} \mathrm{~km}\right)\end{array}$ & $\begin{array}{l}\mathrm{m} \text {, Dipole moment } \\
\qquad\left(10^{22} \mathrm{Am}^{2}\right)\end{array}$ & $\begin{array}{c}\text { Mean } B_{\mathrm{z}}, \bar{B}_{z} . \\
\quad \text { (Gauss) }\end{array}$ & Inclination & $\frac{\bar{B}_{z} / \sqrt{\rho \mu}}{\Omega R_{C}} \times 10^{6}$ & $\Lambda=\frac{\sigma \bar{B}_{z}^{2}}{\rho \Omega}$ \\
\hline Mercury & 58.6 & 2.44 & 1.8 & 0.004 & 0.014 & $5^{\circ}$ & 5.5 & $2 \times 10^{-4}$ \\
\hline Venus & 243 & 6.05 & 3.2 & 0 & - & - & - & - \\
\hline Earth & 1 & 6.37 & 3.48 & 7.9 & 3.7 & $11^{\circ}$ & 13 & 0.2 \\
\hline Mars & 1.03 & 3.39 & 1.8 & 0 & - & - & - & - \\
\hline Jupiter & 0.413 & 69.9 & 55 & 150000 & 18 & $9.6^{\circ}$ & 5.2 & 5 \\
\hline Saturn & 0.440 & 58.2 & 29 & 4600 & 3.8 & $<0.1^{\circ}$ & 2.3 & 0.2 \\
\hline Uranus & 0.718 & 25.3 & $\sim 18$ & 390 & $\sim 1.3$ & $59^{\circ}$ & $\sim 1.0$ & $\sim 10^{-4}$ \\
\hline Neptune & 0.671 & 24.6 & $\sim 20$ & 200 & $\sim 0.5$ & $47^{\circ}$ & $\sim 0.3$ & $\sim 10^{-5}$ \\
\hline
\end{tabular}

One of the most striking features of Table 1 is the surprising uniformity of the normalised magnetic field strengths in the planetary cores,

$\Pi_{B}=\frac{\bar{B}_{z} / \sqrt{\rho \mu}}{\Omega R_{C}}$,

this despite the very different rotation rates, sizes and chemical makeup of the planets. (In Table $1, \Omega$ and $R_{C}$ are the rotation rate and core radius of the planets, $\rho$ and $\sigma$ the mean core density and electrical conductivity, $\mu$ the permeability of free space, and $\lambda=1 / \sigma \mu$.) For example, Mercury is usually regarded as having an anomalously small field, and Jupiter a relatively large field, yet when scaled in this way these two planetary dipoles look remarkably similar. This relative uniformity of $\Pi_{B}$ across the planets has never been adequately explained by the existing scaling laws, most of which have been extrapolated from the numerical dynamos. (Note that, by way of contrast, the Elsasser number, $\Lambda$, varies by a factor of $10^{6}$ across the planets.)

One of the purposes of this study is to try and explain the uniformity of $\Pi_{B}$ (sometimes called the Lorentz number); another is to explore the extent to which the planets and numerical dynamos obey different scaling laws as a result of their different dynamical regimes. To that end we start from first principles, deriving scaling relationships directly from the governing dynamo equations. The dynamo cartoon which guides the process of extracting scaling relationships is a model based on helical waves, first put forward in Davidson (2014) and then in more detail in Davidson \& Ranjan (2015), although we shall show that the resulting scaling equations have much greater generality and would be expected to apply under a broad range of circumstances. These scaling relationships are then applied separately to the numerical dynamos and to the planets, a procedure that highlights both the similarities and the differences between simulations and planets. We show that our proposed scaling relationships explain both the results of the numerical dynamos and the observations of those planets for which we have testable data. In the process we confirm that the scaling laws extracted from the numerical dynamos cannot, in general, be extrapolated to the planets, primarily because viscosity plays a key role in the former, and no role in the latter.

Our scaling analysis is restricted to dipolar fields of the $\alpha^{2}$ type, and so our discussion of the planets is limited to the terrestrial planets and the gas giants, whose magnetic fields are strongly dipolar and roughly aligned with the rotation axis. The ice giants, by contrast, have multipolar fields whose magnetic axes are more equatorially aligned and whose dynamos are likely to be quite different to those of the Earth and Jupiter (Stanley \& Glatzmaier 2010). It is unlikely

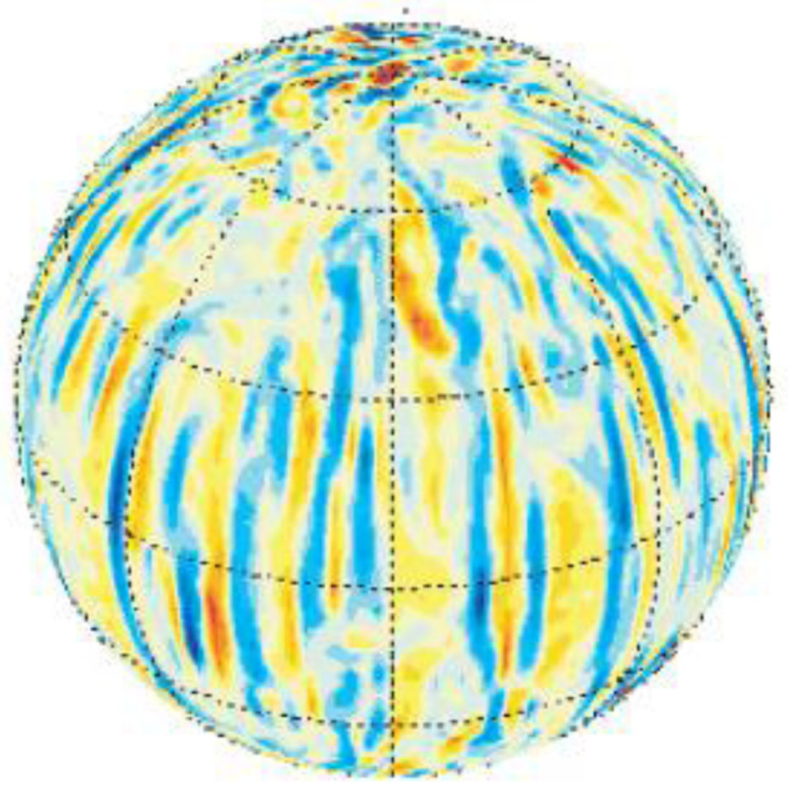

Figure 1. The radial velocity near the mantle in a numerical simulation of the geodynamo (from Christensen \& Wicht 2007).

that our scaling laws apply to the idiosyncratic fields of the ice giants, and so we offer no such comparison.

Before embarking on this scaling analysis, however, we need to summarise how planetary dynamos are thought to operate, at least in some zero-order sense.

\section{PLANETARY DYNAMO CARTOONS}

\subsection{The dynamo cartoon that emerges from the numerical simulations}

The numerical simulations of the geodynamo show that, for the regimes which they can probe, the dynamo is of the $\alpha^{2}$ type and located outside the tangent cylinder, an imaginary cylinder that is concentric with the rotation axis and circumscribes the solid inner core (Olson et al. 1999; Christensen \& Wicht 2007). While there is some evidence of an $\Omega$-effect, this is largely restricted to within the tangent cylinder, driven by local largescale upwellings, and plays no essential role in the dynamo. The dominant flow pattern outside the tangent cylinder consists of long, thin, columnar structures aligned with the rotation axis and within which the flow is highly helical (Fig. 1). This helicity, which is so essential for planetary dynamos, is observed to be negative in the north and positive in the south (Olson 


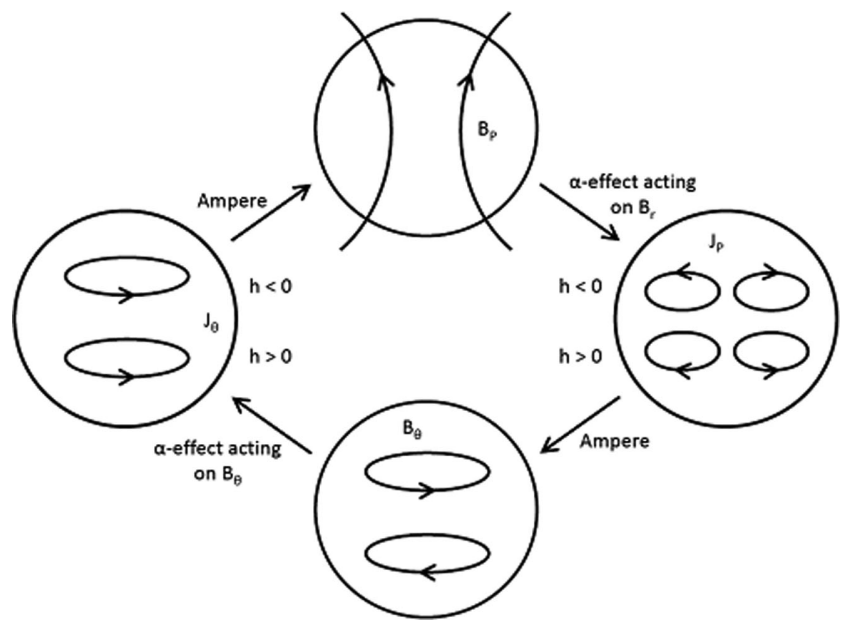

Figure 2. Cartoon of an $\alpha^{2}$-dynamo based on the helicity distribution observed outside the tangent cylinder in most numerical simulations. (From Davidson 2014.)

et al. 1999). Just such an antisymmetric distribution of helicity is, according to traditional mean-field theory, ideal for an $\alpha^{2}$ dynamo, in which the e.m.f. induced by the helical $\alpha$-effect is aligned with the local mean field, $\overline{\mathbf{B}}$, being parallel to $\overline{\mathbf{B}}$ if the helicity is negative and anti-parallel to $\overline{\mathbf{B}}$ if the helicity is positive.

This kind of $\alpha^{2}$ dynamo is most readily described using cylindrical polar coordinates, $(r, \theta, z)$, and it operates, at least in some zero-order sense, in the following way. The columnar vortices interact primarily with the transverse (i.e. radial and azimuthal) components of the magnetic field, spiralling up the transverse field-lines as the helical flow propagates up and down the rotation axis. So, if the dipole points to the north, and the helicity is negative in the north and positive in the south, then the helical convection interacts with the radial component of the dipole magnetic field to produce a radial e.m.f. which is positive in both the north and the south. (Note that, as $B_{r}$ changes sign, so does the helicity.) This then drives a poloidal current density which has a quadruple structure as shown in Fig. 2, being outward in regions of a strong radial e.m.f. and returning at low latitudes where the radial e.m.f. is weaker. The resulting azimuthal magnetic field follows from Ampère's law, and is positive in the north and negative in the south, as seen outside the tangent cylinder in most numerical simulations. (Note that $B_{\theta}$ is often observed to adopt the opposite signs within the tangent cylinder due to a local $\Omega$-effect, but this plays no real part in the dynamo process.) Returning to Fig. 2, the $\alpha$-effect now operates on the east-west field and, given the skew-symmetric helicity distribution, this drives an azimuthal current which is positive in both the north and the south, as required to support the original dipole field.

Of course, the simulations are not planets, and so the geodynamo may be quite different in structure. However, the numerical dynamos are able to reproduce some of the observed features of the Earth's magnetic field (Christensen 2011), and so it seems plausible that the geodynamo is, at least in some zero-order sense, similar to the cartoon in Fig. 2.

\subsection{The search for the source of spatially segregated helicity in planetary dynamos}

One of the key features of this kind of dynamo is the need for large amounts of helicity in the columnar vortices, and for this helicity to be spatially segregated either side of the equatorial plane, say negative in the north and positive in the south, as seen in the simulations. The origin of this helicity, and more crucially the mechanism of its spatial segregation, remains a matter of debate. While Ekman pumping can produce the required asymmetry in helicity, and probably does so in the more viscous and weakly forced of the numerical dynamos, it is extremely unlikely that viscous effects are significant in planets, and in any event there is no mantle on which Ekman layers can form in the gas giants. The fact that, in the more strongly forced numerical dynamos, much of the helicity appears to be internally generated was documented as early as 1999 by Olson et al., and has since been confirmed by other authors (for example, by applying slip boundary conditions). Crucially, however, the physical mechanism by which that helicity is spatially segregated, of one sign in the north and another in the south, has remained largely unexplained. Of course, this spatial segregation is essential to the mean-field dynamo shown in Fig. 2, and so if dynamo action in the planets is be robust, as appears to be the case, then the mechanism by which helicity is spatially segregated must also be robust.

There is, however, one proposed mechanism for the spatial segregation of helicity which is reasonably robust, and this rests on the observation by Olson et al. (1999) and Sakuraba \& Roberts (2009) that the buoyancy flux outside the tangent cylinder tends to be concentrated in and around the equatorial plane. (This equatorial bias in the radial buoyancy flux is thought to be driven by the Lorentz force associated with the zonally averaged poloidal magnetic field and current density, the Lorentz force being azimuthal on the equator and balanced by the mean Coriolis force $2 \Omega\left\langle u_{r}\right\rangle$. See Sakuraba \& Roberts 2009.) The idea, developed in Davidson (2014) and Davidson \& Ranjan (2015), is that the buoyant anomalies in and around the equatorial regions excite helical wave packets, either inertial waves or magnetostrophic waves, and these then propagate up and down the rotation axis towards the mantle. Crucially, upward propagating helical wave packets of this type carry with them negative helicity, while downward propagating wave packets carry positive helicity. Hence this provides a natural way of maintaining negative helicity in the north and positive helicity in the south. This simple idea has been developed into a self-consistent dynamo cartoon in Davidson \& Ranjan (2015), and provides the starting point for this paper.

\section{KINEMATIC PRELIMINARIES}

As a prelude to deriving scaling laws for planetary dynamos we need to estimate the e.m.f. induced by a sea of columnar helical wave-packets, as well as the associated Joule dissipation. In this section, we generalize the analysis of Davidson (2013, 2014) and Davidson \& Ranjan (2015) to estimate the Joule dissipation associated with the helical wave motion. This will enable us to establish a relationship between the Joule dissipation and the mean field in the core, a relationship that provides one of the cornerstones of our scaling analysis. The theoretical ideas summarised below in Section 3.2 are essentially those of Davidson \& Ranjan (2015). These are repeated here partly on the grounds of completeness, but mostly because many of the results detailed in Section 3.2 are subsequently needed in Section 3.3. We use cylindrical polar coordinates $(r, \theta, z)$ throughout and denote the position vector by $\mathbf{x}$. 


\subsection{Integral relationships relating the mean and fluctuating fields}

Our starting point is the integral relationship

$\frac{\mathrm{d}}{\mathrm{d} t} \int_{V_{C}}\left(R_{C}^{2}-\mathbf{x}^{2}\right) \mathbf{B} \mathrm{d} V=2 \int_{V_{C}} \mathbf{x} \times(\mathbf{u} \times \mathbf{B}) \mathrm{d} V-6 \lambda \int_{V_{C}} \mathbf{B} \mathrm{d} V$,

which is obtained by combining the induction equation with (1). (See, for example, the derivation in Davidson 2013.) Of particular interest is the axial component of this equation, which reduces to an evolution equation for the axial field,

$\frac{\mathrm{d}}{\mathrm{d} t} \int_{V_{C}}\left(R_{C}^{2}-\mathbf{x}^{2}\right) B_{z} \mathrm{~d} V=2 \int_{V_{C}} r(\mathbf{u} \times \mathbf{B})_{\theta} \mathrm{d} V-6 \lambda \int_{V_{C}} B_{z} \mathrm{~d} V$.

Evidently, the mean axial field is maintained by the volume integral of $r(\mathbf{u} \times \mathbf{B})_{\theta}$. However, Cowling's theorem tells us that the first integral on the right of (3) is necessarily zero when the velocity and magnetic fields are axisymmetric. So, if we write $\mathbf{B}=\langle\mathbf{B}\rangle(r, z, t)+\mathbf{b}$ and $\mathbf{u}=\langle\mathbf{u}\rangle(r, z, t)+\mathbf{v}$, where $\langle\mathbf{B}\rangle$ and $\langle\mathbf{u}\rangle$ are azimuthal averages of $\mathbf{B}$ and $\mathbf{u}$, then (3) becomes

$\frac{\mathrm{d}}{\mathrm{d} t} \int_{V_{C}}\left(R_{C}^{2}-\mathbf{x}^{2}\right)\left\langle B_{z}\right\rangle \mathrm{d} V=2 \int_{V_{C}} r\langle\mathbf{v} \times \mathbf{b}\rangle_{\theta} \mathrm{d} V-6 \lambda \int_{V_{C}}\left\langle B_{z}\right\rangle \mathrm{d} V$.

This tells us that, in a statistically steady dynamo,

$\langle\mathbf{v} \times \mathbf{b}\rangle_{\theta} \sim \frac{\lambda}{|\mathbf{u}| R_{C}}|\mathbf{u}|\left\langle B_{z}\right\rangle \ll|\mathbf{u}|\left\langle B_{z}\right\rangle$.

Here we may think of $\mathbf{b}$ and $\mathbf{v}$ as being the local fields associated with the columnar eddies, or columnar wave packets, and of $\langle\mathbf{v} \times \mathbf{b}\rangle_{\theta}$ as the mean azimuthal e.m.f. associated with these helical structures via the $\alpha$-effect. Evidently, the perturbation field b is much weaker than the mean dipole field $\left\langle B_{z}\right\rangle$. In Davidson \& Ranjan (2015) a similar integral relationship is derived for the mean east-west field integrated over a hemisphere, say the northern hemisphere, $V_{N}$, and the conclusion for an $\alpha^{2}$-dynamo with negligible $\Omega$-effect is that

$\int_{V_{N}} \frac{z}{r}\langle\mathbf{v} \times \mathbf{b}\rangle_{r} \mathrm{~d} V \sim \lambda \int_{V_{N}}\left(\left\langle B_{\theta}\right\rangle / r\right) \mathrm{d} V$

The same integral expression applies in the southern hemisphere and we conclude that the mean azimuthal field is supported by the radial e.m.f., $\langle\mathbf{v} \times \mathbf{b}\rangle_{r}$, as shown in Fig. 2. Moreover we have

$\langle\mathbf{v} \times \mathbf{b}\rangle_{r} \sim \frac{\lambda}{|\mathbf{u}| R_{C}}|\mathbf{u}|\left\langle B_{\theta}\right\rangle \ll|\mathbf{u}|\left\langle B_{\theta}\right\rangle$.

The primary significance of (5) and (7) for the present study is that $|\mathbf{b}| \ll\langle|\mathbf{B}|\rangle$, a situation known as first-order smoothing in mean-field theory.

\subsection{The $\alpha$-effect driven by helical, columnar vortices or wave packets}

We now take advantage of the fact that $|\mathbf{b}| \ll\langle|\mathbf{B}|\rangle$ to evaluate the induced e.m.f. and Joule dissipation associated with the $\alpha$-effect. We start with the induced e.m.f., leaving the Joule dissipation until Section 3.3. Following Davidson \& Ranjan (2015), it is assumed that the small-scale motion outside the tangent cylinder is columnar and helical, perhaps a collection of convection rolls, or else a sea of inertial or magnetostrophic wave packets. Such wave packets would be generated primarily in regions where the buoyancy field is strong, such as near the equatorial plane, and then carry negative helicity to the north and positive helicity to the south as they propagate up and down the rotation axis. It is also assumed that a typical transverse dimension of the columnar eddies, $\delta$, is much less than the core radius, $R_{C}$, so that a local mean field, $\langle\mathbf{B}\rangle$, varies slowly on the scale of $\delta$ and may be treated as locally uniform.

Since the analysis is essentially local, we may now think of $\langle\mathbf{B}\rangle$ as representing a local volume average of $\mathbf{B}$, rather than an azimuthal average. It is also convenient to adopt local Cartesian coordinates with $z$ aligned with the rotation axis and $\langle\mathbf{B}\rangle$ with the $x$-axis. We then make the following additional approximations:

(i) axial gradients in $\mathbf{v}$ are small, so that $\mathbf{v} \approx \mathbf{v}(x, y)$;

(ii) the fluctuations in velocity have maximal helicity, as would be the case for monochromatic inertial or magnetostrophic waves, with $\mathbf{v}=\delta \boldsymbol{\omega}=\delta \nabla \times \mathbf{v}$, where $\delta$ is a positive or negative constant having the dimensions of length;

(iii) the fluctuations can be treated as statistically homogeneous, as well as statistically symmetric about the component of the local mean field normal to the rotation axis, $\left\langle\mathbf{B}_{\perp}\right\rangle=\langle\mathbf{B}\rangle-\left\langle\mathbf{B}_{z}\right\rangle$.

In Davidson \& Ranjan (2015) the validity of these various assumptions are tested for the particular case of a sea of inertial wave packets generated by random buoyant anomalies located near the equator. It turns out that they are a reasonable approximation.

Given these assumptions, the starting point for the analysis is Ohm's law written as

$\frac{\partial \mathbf{A}}{\partial t}=\mathbf{u} \times \mathbf{B}-\nabla \Phi-\lambda \nabla \times \mathbf{B}$,

where $\mathbf{A}$ is the (solenoidal) vector potential for $\mathbf{B}$ and we have substituted for $\mathbf{E}$ using Faraday's law. We now decompose all quantities into a local spatial mean and the perturbation about that mean: $\mathbf{B}=\langle\mathbf{B}\rangle+\mathbf{b}, \mathbf{u}=\langle\mathbf{u}\rangle+\mathbf{v}, \mathbf{A}=\langle\mathbf{A}\rangle+\mathbf{a}$ and $\Phi=\langle\Phi\rangle+\varphi$. Applying first-order smoothing to the fluctuating part of (8) then yields

$\frac{\partial \mathbf{a}}{\partial t}=\mathbf{v} \times\langle\mathbf{B}\rangle-\nabla \varphi-\lambda \nabla \times \mathbf{b}$.

It is convenient at this point to introduce a vector potential for the velocity field, $\mathbf{v}$, defined by $\mathbf{v}=\nabla \times \mathbf{c}, \nabla \cdot \mathbf{c}=0$. The scalar potential $\varphi$ is then governed by the divergence of (9) in the form $\nabla^{2} \varphi=-\nabla^{2}(\langle\mathbf{B}\rangle \cdot \mathbf{c})$. It follows that $\varphi=-\langle\mathbf{B}\rangle \cdot \mathbf{c}+\hat{\varphi}$, where $\nabla^{2} \hat{\varphi}=0$.

Next we invoke assumption (ii) in the form $\mathbf{c}=\delta \mathbf{v}=\delta^{2} \boldsymbol{\omega}$. It follows immediately that (9) may be rewritten as

$\left[\partial / \partial t-\lambda \nabla^{2}\right](\mathbf{a}-\delta \mathbf{b})=0$,

and so, as noted in Davidson \& Ranjan (2015), if $\mathbf{v}$ has maximal helicity, then so eventually does $\mathbf{b}$. That is, irrespective of the initial conditions, $\mathbf{b}$ will become progressively more helical with time. Thus we shall take $\mathbf{a}=\delta \mathbf{b}$, which in any event holds for both inertial and magnetostrophic waves. In this case our governing eq. (9) becomes

$\frac{\partial \mathbf{b}}{\partial t}+\frac{\lambda}{\delta^{2}} \mathbf{b}=\frac{1}{\delta}[\mathbf{v} \times\langle\mathbf{B}\rangle-\nabla \varphi]$,

from which

$\mathbf{v} \times \mathbf{b}+\frac{\delta^{2}}{\lambda} \mathbf{v} \times \frac{\partial \mathbf{b}}{\partial t}=\frac{\delta}{\lambda}[\mathbf{v} \times(\mathbf{v} \times\langle\mathbf{B}\rangle)+\nabla \times(\varphi \mathbf{v})-\varphi \nabla \times \mathbf{v}]$. 
If we now assume statistical homogeneity we have

$\langle\varphi \boldsymbol{\omega}\rangle=-\langle(\langle\mathbf{B}\rangle \cdot \mathbf{c}) \boldsymbol{\omega}\rangle=-\langle(\langle\mathbf{B}\rangle \cdot \mathbf{v}) \mathbf{v}\rangle$,

and so (12) averages to give

$\langle\mathbf{v} \times \mathbf{b}\rangle+\frac{\delta^{2}}{\lambda}\left\langle\mathbf{v} \times \frac{\partial \mathbf{b}}{\partial t}\right\rangle=-\frac{\delta}{\lambda}\left\langle\mathbf{v}^{2}\langle\mathbf{B}\rangle-2(\mathbf{v} \cdot\langle\mathbf{B}\rangle) \mathbf{v}\right\rangle$,

(Davidson \& Ranjan 2015).

Finally we deploy assumption (i) in the form $\mathbf{v}=\mathbf{v}(x, y)=\delta \boldsymbol{\omega}$, and again invoke statistical homogeneity. This yields

$\left\langle v_{x} v_{z}\right\rangle=\left\langle v_{y} v_{z}\right\rangle=0, \quad\left\langle v_{z}^{2}\right\rangle=\left\langle v_{x}^{2}+v_{y}^{2}\right\rangle$,

while statistical symmetry about the local mean field $\left\langle\mathbf{B}_{\perp}\right\rangle$ demands $\left\langle v_{x} v_{y}\right\rangle=0$. Eq. (14) now simplifies to

$\langle\mathbf{v} \times \mathbf{b}\rangle+\frac{\delta^{2}}{\lambda}\left\langle\mathbf{v} \times \frac{\partial \mathbf{b}}{\partial t}\right\rangle=-\frac{2 \delta}{\lambda}\left\langle v_{y}^{2}\right\rangle\left\langle\mathbf{B}_{\perp}\right\rangle$,

which is the key result of this section.

Perhaps some comments are in order at this point. First, there is no mean e.m.f. in the axial direction. Second, so far the analysis applies equally to strongly helical columnar vortices and columnar wave packets, as we have not had to assume any wave-like properties. Third, we have made no assumption about the size of the magnetic Reynolds number, $u \delta / \lambda$. Fourth, the columnar structures could be either tube like, with only one characteristic transverse dimension, or (more likely) sheet-like, with two characteristic transverse dimensions (Davidson \& Ranjan 2015). In the latter case, $\delta$ will be the smaller of those two transverse dimensions.

\subsection{Estimating the Joule dissipation associated with helical wave packets}

Let us now calculate the Joule dissipation caused by the passage of the columnar vortices or wave packets through the mean field. We start by rewriting (11) in the form

$\mathbf{j}+\frac{\delta^{2}}{\lambda} \frac{\partial \mathbf{j}}{\partial t}=\sigma[\mathbf{v} \times\langle\mathbf{B}\rangle-\nabla \varphi]$,

from which we find,

$\mathbf{j}^{2}+\frac{\delta^{2}}{\lambda} \frac{\partial}{\partial t}\left(\mathbf{j}^{2} / 2\right)=\frac{\sigma}{\delta \mu}[\mathbf{b} \cdot(\mathbf{v} \times\langle\mathbf{B}\rangle)-\nabla \cdot(\varphi \mathbf{b})]$,

and

$$
\begin{aligned}
& \left(\frac{\delta^{2}}{\lambda} \frac{\partial \mathbf{j}}{\partial t}\right)^{2}+\frac{\delta^{2}}{\lambda} \frac{\partial}{\partial t}\left(\mathbf{j}^{2} / 2\right) \\
& \quad=\frac{\sigma}{\delta \mu} \frac{\delta^{2}}{\lambda}\left[\frac{\partial \mathbf{b}}{\partial t} \cdot(\mathbf{v} \times\langle\mathbf{B}\rangle)-\nabla \cdot\left(\varphi \frac{\partial \mathbf{b}}{\partial t}\right)\right] .
\end{aligned}
$$

Adding and averaging (18) and (19) yields

$\frac{\delta \mu}{\sigma}\left\langle\left(\mathbf{j}+\frac{\delta^{2}}{\lambda} \frac{\partial \mathbf{j}}{\partial t}\right)^{2}\right\rangle=\frac{\delta^{2}}{\lambda}\left\langle\frac{\partial \mathbf{b}}{\partial t} \cdot(\mathbf{v} \times\langle\mathbf{B}\rangle)\right\rangle+\langle\mathbf{b} \cdot(\mathbf{v} \times\langle\mathbf{B}\rangle)\rangle$,

or equivalently,

$\frac{\delta \mu}{\sigma}\left\langle\left(\mathbf{j}+\frac{\delta^{2}}{\lambda} \frac{\partial \mathbf{j}}{\partial t}\right)^{2}\right\rangle=-\left[\langle\mathbf{v} \times \mathbf{b}\rangle+\frac{\delta^{2}}{\lambda}\left\langle\mathbf{v} \times \frac{\partial \mathbf{b}}{\partial t}\right\rangle\right] \cdot\langle\mathbf{B}\rangle$.

Finally, comparing (16) and (20) gives the remarkably simple result

$\frac{\mu}{\sigma}\left\langle\left(\mathbf{j}+\frac{\delta^{2}}{\lambda} \frac{\partial \mathbf{j}}{\partial t}\right)^{2}\right\rangle=\frac{2}{\lambda}\left\langle v_{y}^{2}\right\rangle\left\langle\mathbf{B}_{\perp}^{2}\right\rangle$.
Let us now restrict the analysis to helical waves (inertial or magnetostrophic waves), so that that we can fix the relative phases of $\mathbf{b}$ and $\partial \mathbf{b} / \partial t$ in (16), and of $\mathbf{j}$ and $\partial \mathbf{j} / \partial t$ in (21). In particular we take $\mathbf{v}$ and $\mathbf{b}$ to have the circularly polarized form characteristic of an inertial or magnetostrophic wave:

$\mathbf{v}(x, y, t)=v_{0}\left(\mathrm{i} \delta k_{y},-\mathrm{i} \delta k_{x}, 1\right) \exp [\mathrm{i}(\mathbf{k} \cdot \mathbf{x}-\varpi t)]$,

$\mathbf{b}(x, y, t)=b_{0}\left(\mathrm{i} \delta k_{y},-\mathrm{i} \delta k_{x}, 1\right) \exp [\mathrm{i}(\mathbf{k} \cdot \mathbf{x}-\varpi t)]$,

with $k_{x}^{2}+k_{y}^{2}=\delta^{-2}$ and the real part is understood. The curl of (11) then requires that $v_{0}$ and $b_{0}$ are related according to

$\left[1+\frac{\mathrm{i} \lambda}{\delta^{2} \varpi}\right] \varpi b_{0}=-\left\langle\mathbf{B}_{\perp}\right\rangle \cdot \mathbf{k} v_{0}$.

After a little algebra our expressions for the induced e.m.f. and Joule dissipation, (16) and (21), then simplify to

$$
\begin{aligned}
& \langle\mathbf{v} \times \mathbf{b}\rangle=-\frac{2 \delta}{\lambda} \frac{\left\langle v_{y}^{2}\right\rangle\left\langle\mathbf{B}_{\perp}\right\rangle}{1+\left(\varpi \delta^{2} / \lambda\right)^{2}}, \\
& \frac{\mu}{\sigma}\left\langle\mathbf{j}^{2}\right\rangle=-\delta^{-1}\langle\mathbf{v} \times \mathbf{b}\rangle \cdot\left\langle\mathbf{B}_{\perp}\right\rangle=\frac{2}{\lambda} \frac{\left\langle v_{y}^{2}\right\rangle\left\langle\mathbf{B}_{\perp}^{2}\right\rangle}{1+\left(\varpi \delta^{2} / \lambda\right)^{2}} .
\end{aligned}
$$

Expression (24) is the primary result of our kinematic analysis. Note that if the columnar wave packets are sheet-like, elongated in the direction of $\mathbf{B}_{\perp}$, then $\left\langle v_{y}^{2}\right\rangle \ll\left\langle\mathbf{v}^{2}\right\rangle$.

\section{RELATING THE JOULE DISSIPATION AND BUOYANCY TO THE MEAN B - F I E L D}

We are finally in a position to relate the Joule dissipation to the mean field strength in the core, a relationship which forms one of the cornerstones of our scaling analysis. From (24) we see that the average Joule dissipation per unit mass is related to the induced e.m.f. by

$\frac{\left\langle\mathbf{j}^{2}\right\rangle}{\sigma \rho}=-\frac{\langle\mathbf{v} \times \mathbf{b}\rangle \cdot\left\langle\mathbf{B}_{\perp}\right\rangle}{\delta \mu \rho}$.

For a statically steady dynamo this dissipation must equal the mean rate of working of the buoyancy forces per unit mass (Christensen et al. 2009), which we label as P. Moreover, for a steady dynamo the integral relationship (4) yields,

$\int_{V_{C}} r\langle\mathbf{v} \times \mathbf{b}\rangle_{\theta} \mathrm{d} V=3 \lambda \int_{V_{C}}\left\langle B_{z}\right\rangle \mathrm{d} V$,

and combining these various expressions we obtain

$P \sim \frac{\left\langle\mathbf{j}^{2}\right\rangle}{\sigma \rho} \sim \frac{\lambda}{\delta R_{C}} \frac{\left\langle\mathbf{B}^{2}\right\rangle}{\rho \mu}$.

Introducing the notation

$v_{a} \sim \frac{\left\langle\mathbf{B}^{2}\right\rangle^{1 / 2}}{\sqrt{\rho \mu}} \sim \frac{B_{r m s}}{\sqrt{\rho \mu}}, \quad v_{P} \sim\left(P R_{C}\right)^{1 / 3}$, 
for the characteristic Alfvén speed in the core and for a velocity scale associated with the rate of working of the buoyancy force, our kinematic analysis reduces to the remarkably simple estimate

$\frac{v_{a}^{2} \lambda}{\delta} \sim v_{P}^{3}$

This is all we need take away from Sections 3 and 4. Note that this relationship is independent of the value of the magnetic Reynolds number at the scale of $\delta$. Note also that if the columnar wave-packets are sheet-like, with two characteristic dimensions in the transverse plane, $\delta$ will correspond to the smaller of those two dimensions.

Finally we observe that (28) has a generality well beyond that of helical-wave dynamos, and may apply to a broad range of dipolar, $\alpha^{2}$ dynamos. The point is that (28) rests simply on the assumption that the dynamo is of the $\alpha^{2}$ type and on (25) and (26), the latter of which is valid quite generally. However (25) holds, at least approximately, whenever the small-scale current is of order $\mathbf{j} \sim \sigma \mathbf{v} \times\left\langle\mathbf{B}_{\perp}\right\rangle$, a situation which is likely to arise even in dynamos driven by, say, Ekman pumping.

\section{SCALING LAWS FOR PLANETARY DYNAMOS}

We now supplement (28) by an order-of-magnitude force balance, which then provides the governing scaling equations for planetary dynamos. We shall see that this leads to two distinct sets of scaling laws, one for the numerical dynamos and one for the planets.

\subsection{General scaling equations for both the numerical dynamos and the planets}

Suppose that, for simplicity, we take the fluid in the conducting core to be Boussinesq, governed by

$\frac{\partial \mathbf{u}}{\partial t}=2 \mathbf{u} \times \boldsymbol{\Omega}-\nabla(p / \rho)+\vartheta \mathbf{g}+\rho^{-1} \mathbf{J} \times \mathbf{B}$,

where $\mathbf{g}$ is the gravitational acceleration (which is anti-parallel to $\mathbf{x}$ ), $\vartheta=\rho^{\prime} / \rho, \rho^{\prime}$ is the perturbation in density, and $\rho$ the mean density. Note that the viscous and non-linear inertial terms are omitted in (29), since they are irrelevant in the core of the Earth, where the Rossby number based on $R_{C}$ is around $10^{-6}$ and the Ekman number is of order $10^{-15}$. The equivalent vorticity equation is, of course.

$\frac{\partial \boldsymbol{\omega}}{\partial t}=2 \boldsymbol{\Omega} \cdot \nabla \mathbf{u}+\nabla \vartheta \times \mathbf{g}+\rho^{-1} \nabla \times(\mathbf{J} \times \mathbf{B})$,

while the rate of working of the buoyancy force per unit mass is

$P=\vartheta \mathbf{g} \cdot \mathbf{u}$.

Traditional scaling analyses for planetary dynamos tend to start with the assumption that the Elsasser number is of order unity, $\Lambda=\sigma B^{2} / \rho \Omega \sim 1$, which is based on the idea that the Lorentz force, which is taken to be of order $|\mathbf{j} \times \mathbf{B}| \sim \sigma u B^{2}$, is of the same order of magnitude as the Coriolis force, $2 \rho \mathbf{u} \times \boldsymbol{\Omega}$. However, as noted by several authors (e.g. Davidson 2014), this is too simplistic for rapid rotators like the Earth and the gas giants, where the flow is anisotropic with typical flow structures highly elongated along the rotation axis. In such cases the curl and divergence of the Coriolis force are

$\nabla \times(\mathbf{u} \times \boldsymbol{\Omega})=(\boldsymbol{\Omega} \cdot \nabla) \mathbf{u} \sim \frac{\Omega u}{R_{C}}$, $\nabla \cdot(\mathbf{u} \times \boldsymbol{\Omega})=\boldsymbol{\Omega} \cdot \nabla \times \mathbf{u} \sim \frac{\Omega u}{\delta}$,

so that the curl of the Coriolis force is much smaller than its divergence. Consequently, if we perform a Helmholtz decomposition on the Coriolis force, dividing it into irrotational and solenoidalrotational parts, the dominant term is the former. Moreover, the irrotational part of the Coriolis force is simply balanced by pressure gradients (the quasi-geostrophic force balance) and so the Lorentz force, if order one, should be balanced against the much weaker rotational component.

A second problem is that (24) yields $|\mathbf{j} \times \mathbf{B}| \sim \sigma\left\langle v_{y}^{2}\right\rangle^{1 / 2} B^{2}$ which, for sheet-like vortices, is much smaller than the usual estimate of $|\mathbf{j} \times \mathbf{B}| \sim \sigma\left\langle\mathbf{v}^{2}\right\rangle^{1 / 2} B^{2} \sim \sigma u B^{2}$. When a more careful force balance is performed, balancing the curl of $|\mathbf{j} \times \mathbf{B}| \sim \sigma\left\langle v_{y}^{2}\right\rangle^{1 / 2} B^{2}$ against $\Omega u / R_{C}$, we find no reason to suppose that $\Lambda \sim 1$, and indeed we have the possibility that $\Lambda \ll 1$, or even $\Lambda \gg 1$. So the suggestion that $\Lambda \sim 1$ lacks theoretical support.

An alternative approach is to assume that the curl of the buoyancy force is in approximate balance with the curl of the Coriolis force. In such a case (30) and (31) yield

$\frac{\Omega u}{R_{C}} \sim \frac{\vartheta g}{\delta} \sim \frac{P}{\delta u}$

or equivalently

$\Omega \delta u^{2} \sim P R_{C} \sim v_{P}^{3}$.

Since (28) is, in some sense, equivalent to equating the curl of the Lorentz force to the curl of the buoyancy force, we conclude that our scaling equations for planetary dynamos driven by helical waves (either inertial or magnetostrophic waves) are simply

$v_{P}^{3} \sim \frac{v_{a}^{2} \lambda}{\delta} \sim \Omega \delta u^{2}$.

Moreover, since (28) has a generality beyond that of helical-wave dynamos, then so does (36). (Actually (36) was derived in Davidson 2014 , although the line of reasoning used here is somewhat different and subject to less restrictions.)

Note that $\mathrm{P}$ and $R_{C}$ do not appear explicitly in (36), but rather indirectly through the characteristic velocity $v_{P}$. Note also that the fluid viscosity does not appear in (36), as must be the case because viscous stresses are irrelevant in the core a planet (though not in the numerical dynamos). Finally recall that, in the case of a helical-wave dynamo, (36) is independent of the value of the magnetic Reynolds number at the scale of $\delta$, and of the characteristic cross-sectional shape of the columnar wave-packets, which may be sheet-like or tube-like.

In cases where the buoyancy flux is dominated by thermal buoyancy, we may estimate $\mathrm{P}$, and hence $v_{P}$, from the convective heat flux through the core. In particular, if $\beta$ is the expansion coefficient, and $T^{\prime}$ the temperature perturbation, then the rate of working of the thermal buoyancy force per unit mass is $P=-\beta \overline{T^{\prime} \mathbf{u}} \cdot \mathbf{g}$. In such cases $\mathrm{P}$ can be expressed in terms of the time-averaged convective heat flux per unit area, $\dot{\mathbf{q}}=\rho c_{p} \overline{T^{\prime} \mathbf{u}}$, according to $P=\left(g \beta / \rho c_{p}\right) \dot{q}$. (See, for example, Christensen et al. 2009). Reasonable estimates of $\dot{q}$ are available for the Earth, Jupiter and Saturn. For example, for Jupiter and Saturn we have $\dot{q} \approx 5.7$ and $2.0 \mathrm{~W} \mathrm{~m}^{-2}$, respectively (Read et al. 2015), and using estimates of $\beta / c_{p}$ from Christensen $\&$ Aubert (2006), we find $P \approx 1.2 \times 10^{-10} \mathrm{~m}^{2} \mathrm{~s}^{-3}$ for Jupiter and $P \approx 0.43 \times 10^{-10} \mathrm{~m}^{2} \mathrm{~s}^{-3}$ for Saturn. 
In situations where the compositional buoyancy makes a significant contribution to the buoyancy flux, as in the Earth, a common strategy is to estimate $\mathrm{P}$ based on $P=\left(g \beta / \rho c_{p}\right) \dot{q}$ and to then inflate $\mathrm{P}$ according to the expected ratio of compositional to thermal buoyancy. In the case of the Earth, for example, the thermal and compositional buoyancy are thought to be of similar strengths (Roberts \& King 2013), so it seems plausible to double the value $P=\left(g \beta / \rho c_{p}\right) \dot{q}$ to get an estimate of the total rate of working of the buoyancy force. Taking the characteristic convective heat flux to be around 3TW ( $\dot{q} \approx 0.02 \mathrm{~W} \mathrm{~m}^{-2}$ ), and doubling this to accommodate compositional buoyancy, yields $P \approx 5.1 \times 10^{-13} \mathrm{~m}^{2} \mathrm{~s}^{-3}$ for the Earth.

So we may regard $\mathrm{P}$, and hence $v_{P}$, as prescribed. The velocities $v_{a}$ and $u$, on the other hand, are dependent quantities, to be derived from (36) once $\delta$ has been determined. One complication here is that it turns out that the way in which $\delta$ is set in the numerical dynamos is very different to the way it is fixed in the planets, and so quite distinct scaling laws emerge for the numerical simulations and real planetary dynamos, though as we shall see, both probably satisfy (36).

Note that (36) estimates the net energy density (magnetic plus kinetic) to be of order

$e \sim v_{a}^{2}+u^{2} \sim v_{P}^{3}\left[\frac{\delta}{\lambda}+\frac{1}{\Omega \delta}\right]$.

For a given value of $\mathrm{P}$, or core heat flux, this has a minimum at $\delta_{\min } \sim \sqrt{\lambda / \Omega}$, which corresponds to an equipartition between magnetic and kinetic energy. More generally, the ratio of magnetic to kinetic energy is, according to (36),

$\frac{v_{a}^{2}}{u^{2}} \sim \frac{\Omega \delta^{2}}{\lambda}$.

Since the magnetic energy is thought to be dominant in planetary dynamos, this tells us that $\delta$ must exceed $\delta_{\min }, \delta>\sqrt{\lambda / \Omega}$.

Notice also that dipolar dynamos of the type observed in the Earth and gas giants require that the small-scale Rossby number, $\operatorname{Ro}_{\delta}=u / \Omega \delta$, is smaller than order one. This was first observed empirically in the numerical simulations of Christensen \& Aubert (2006). However, it almost certainly has its origins in the fact that inertial waves cannot propagate when $\mathrm{Ro}_{\delta}$ exceeds a value of around 0.4 (see, for example, Bin Baqui \& Davidson 2015, where $\delta$ is defined as the transverse integral scale), yet translating columnar vortices rely on inertial wave packets to maintain their quasi-geostrophy (Davidson \& Ranjan 2015). This interpretation is given weight by the observation that the loss of the dipolar field more or less coincides with the loss of columnar flow structures. In any event, dipolar dynamos cannot be maintained when $\operatorname{Ro}_{\delta}$ exceeds $u / \Omega \delta \sim 0.4$, and from (36) this corresponds to

$\operatorname{Ro}_{\delta} \sim\left(u / v_{P}\right)^{3} \sim 0.4$.

Evidently, if our scaling laws are to be consistent with the dipolar fields observed in the Earth and the gas giants, we are restricted to the range $\operatorname{Ro}_{\delta} \leq O(1)$ and $u \leq O\left(v_{P}\right)$. More generally, we may rewrite (36) in terms of $\operatorname{Ro}_{\delta}=u / \Omega \delta$ as

$\frac{u}{v_{P}} \sim\left(\operatorname{Ro}_{\delta}\right)^{1 / 3}, \quad \frac{v_{a}}{v_{P}} \sim \frac{v_{P}}{\sqrt{\lambda \Omega}}\left(\operatorname{Ro}_{\delta}\right)^{-1 / 3}$,

which places an upper bound on the convective velocity of $u \leq$ $O\left(v_{P}\right)$, and a lower bound on the rms magnetic field of $v_{a} \geq$ $O\left(v_{P}^{2} / \sqrt{\lambda \Omega}\right)$. These are the key scaling relationships to emerge from our analysis. They apply equally to the dipolar numerical dynamos and dipolar $\alpha^{2}$ planetary dynamos, provided that the estimate $\mathbf{j} \sim \sigma \mathbf{v} \times\left\langle\mathbf{B}_{\perp}\right\rangle$ holds true in both cases.

The weakness of (40) is that $\delta$ remains undetermined, and it is only once we know how $\delta$ scales that true scaling laws can emerge for $u$ and $v_{a}$. As we shall see, the way in which $\delta$ is set in the numerical dynamos is different to the way it is determined in the planets, so these two sets of dynamos obey distinct scaling laws. Moreover, it is entirely possible that the process of setting $\delta$ in, say, the gas giants, is quite different to that in the terrestrial planets, in which case the hypothesis that there exists universal scaling laws for $\alpha^{2}$ planetary dynamos fails. Never-the-less, many authors have proposed such universal scaling laws, so we shall test these against (36), or equivalently (40), and against the observational data.

\subsection{Scaling laws for the numerical dynamos}

It is still unclear what fixes the unknown length-scale $\delta$ in planetary cores, though we shall discuss a variety of possibilities in the next section. However, the numerical dynamos are more viscous than the planets by a factor of $\sim 10^{9}$, as measured by the Ekman number, $\mathrm{Ek}=v / \Omega R_{C}^{2}$. So in the simulations it is likely that $\delta$ is set by viscous forces, which demands $\delta / R_{C} \sim \mathrm{Ek}^{1 / 3}$ (King \& Buffett 2013). In such cases, the scaling laws corresponding to (36) become

$\Pi_{B} \sim \Pi_{P}{ }^{1 / 2} \operatorname{Pr}_{\mathrm{m}}{ }^{1 / 2} \mathrm{Ek}^{-1 / 3}, \quad$ Ro $\sim \Pi_{P}{ }^{1 / 2} \mathrm{Ek}^{-1 / 6}$,

where

$$
\begin{gathered}
\Pi_{p}=\frac{P}{\Omega^{3} R_{C}^{2}}=\left(\frac{v_{P}}{\Omega R_{C}}\right)^{3}, \quad \Pi_{B}=\frac{B_{r m s} / \sqrt{\rho \mu}}{\Omega R_{C}}, \\
\operatorname{Ro}=\frac{u}{\Omega R_{C}}, \quad \operatorname{Pr}_{\mathrm{m}}=\frac{v}{\lambda} .
\end{gathered}
$$

Scaling (41b) for Ro has been proposed by several authors (see, for example, King \& Buffett 2013 or Davidson 2014) and found to be a good match to data sets for the numerical dynamos. On the other hand, (41a) for $\Pi_{B}$ was first proposed in Davidson (2014), though in that paper the scaling analysis was limited to helical-wave dynamos with a low magnetic Reynolds number on the scale of $\delta$, whereas here we suggest that (41a) has much greater generality. Note that $v$ and $R_{C}$ both appear explicitly in these viscous scalings, despite their absence in (36).

Let us turn now to the assertion that $\operatorname{Ro}_{\delta}=u / \Omega \delta \approx 0.4$ represents the transition from dipolar to multipolar dynamos. Combining Ro $\sim \Pi_{P}{ }^{1 / 2} \mathrm{Ek}^{-1 / 6}$ with $\delta / R_{C} \sim \mathrm{Ek}^{1 / 3}$, our scaling analysis predicts $\operatorname{Ro}_{\delta} \sim \Pi_{P}{ }^{1 / 2} \mathrm{Ek}^{-1 / 2}$. To fix thoughts let us take the pre-factor in this estimate to be unity: $\mathrm{Ro}_{\delta}=\Pi_{P}{ }^{1 / 2} \mathrm{Ek}^{-1 / 2}$. When discussing the numerical dynamos it has become conventional to replace $\Pi_{P}$ and Ek by the related parameters $\operatorname{Ra}_{Q}=\Pi_{P} / r(1-r)^{2}$ and $E=\mathrm{Ek} /(1-r)^{2}$, where $r$ is the ratio of the radii of the inner and outer cores. For the Earth $\mathrm{Ra}_{Q}=6.76 \Pi_{P}$. Combining $\mathrm{Ro}_{\delta}=$ $\Pi_{P}{ }^{1 / 2} \mathrm{Ek}^{-1 / 2}$ with the transition criterion $\mathrm{Ro}_{\delta}=u / \Omega \delta \approx 0.4$, and replacing $\Pi_{P}$ and Ek by $\mathrm{Ra}_{Q}$ and $E$ with an Earth-like value of $r$, we conclude that the transition from dipolar to multipolar dynamos in the numerical simulations should occur at $\mathrm{Ra}_{Q} / E \approx 0.5$.

Fig. 3 shows the results of the suite of numerical dynamos tabulated in Christensen \& Aubert (2006). In particular, $f_{\text {dip }}$ is plotted as a function of $2 \mathrm{Ra}_{Q} / E$, where $f_{\text {dip }}$ is the field strength of the dipole at the outer boundary of the simulation, divided by the field contained in the harmonics of degree 1 to 12. It is conventional to regard multipolar and dipolar cases as corresponding to $f_{\text {dip }}<0.35$ and $f_{\text {dip }}>0.5$, respectively. Evidently the transition criterion $2 \mathrm{Ra}_{Q} / E \approx 1$ does a 


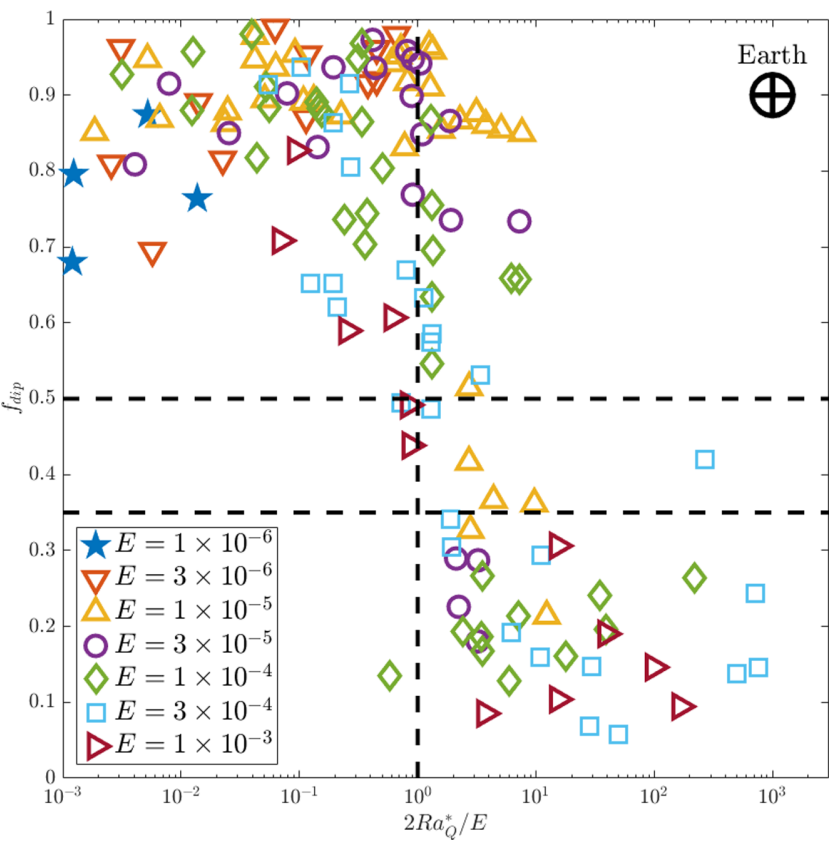

Figure 3. $f_{\text {dip }}$ plotted as a function of $2 \mathrm{Ra}_{Q} / E$. (The data is from Christensen \& Aubert 2006.)

reasonable job of distinguishing between dipolar and non-dipolar cases.

Scaling laws $(41 \mathrm{a}, \mathrm{b})$ are compared with the same data set in Fig. 4. (The comparison for Ro is already given in King \& Buffett 2013 , and we present it here simply for reference. The more important comparison is that for $\Pi_{B}$.) In order to allow for the viscous dissipation in the numerical dynamos, which has been ignored in our scaling analysis, we follow Christensen \& Aubert (2006) and replace $\Pi_{B}$ by $\Pi_{B} / \sqrt{f_{\text {ohm }}}$, where $f_{\text {ohm }}$ is the ratio of the Joule dissipation to the total dissipation. The comparison looks favourable and so it seems that the numerical dynamos offer considerable support for the scaling laws given by (41), and by implication, support for (36).

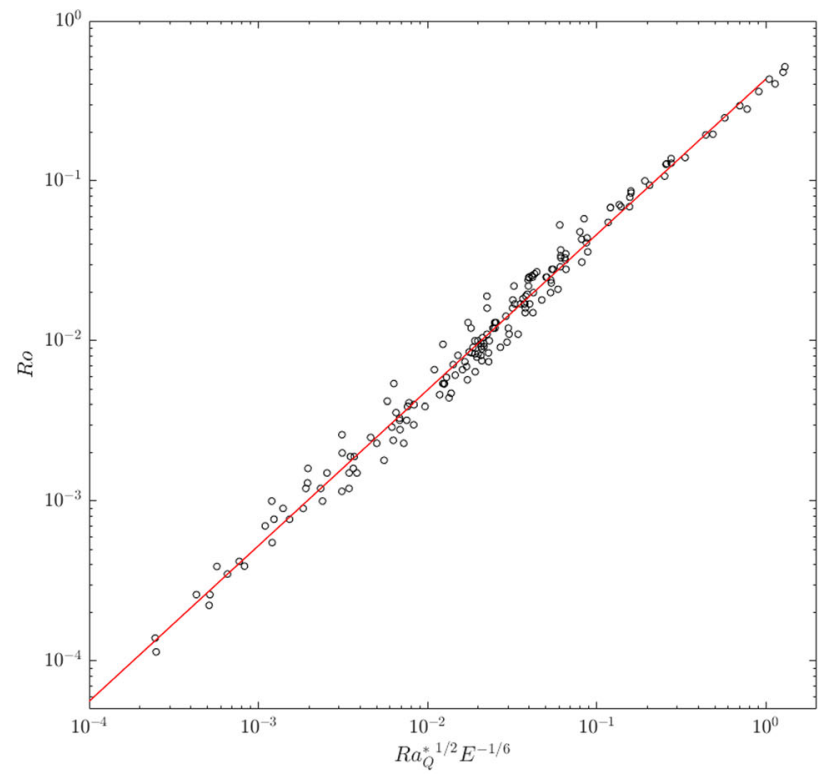

Finally we note that (41) may rewritten in the form

$$
\begin{aligned}
& \Pi_{B} \sim \Pi_{P}{ }^{7 / 18} \operatorname{Pr}_{m}{ }^{1 / 6}\left(v_{P} R_{C} / \lambda\right)^{1 / 3}, \\
& \operatorname{Ro} \sim \Pi_{P}{ }^{4 / 9} \operatorname{Pr}_{m}{ }^{-1 / 6}\left(v_{P} R_{C} / \lambda\right)^{1 / 6} .
\end{aligned}
$$

As noted in Davidson (2014), the quantity $\left(v_{P} R_{C} / \lambda\right)^{1 / 3}$ is usually of order 10 in the numerical dynamos, invariably lying in the range $5 \rightarrow 15$. For such limited data sets, these viscous scaling laws reduce to the deceptively simple expressions $\Pi_{B} \sim \Pi_{P}{ }^{7 / 18} \operatorname{Pr}_{m}{ }^{1 / 6}$ and Ro $\sim \Pi_{P}{ }^{4 / 9} \operatorname{Pr}_{m}{ }^{-1 / 6}$. These, in turn, are rather close to the empirical scalings $\Pi_{B} \sim \Pi_{P}{ }^{0.31} \operatorname{Pr}_{m}{ }^{0.16}$ and $\mathrm{Ro} \sim \Pi_{P}{ }^{0.44} \operatorname{Pr}_{m}{ }^{-0.13}$, proposed by Stelzer \& Jackson (2013), and also $\Pi_{B} \sim \Pi_{P}{ }^{0.32} \operatorname{Pr}_{m}{ }^{0.11}$ and Ro $\sim \Pi_{P}{ }^{0.43} \operatorname{Pr}_{m}{ }^{-0.13}$, suggested earlier by Christensen \& Aubert (2006). (Both sets of empirical scaling laws were derived from more or less the same suite of numerical dynamos.)

\subsection{Scaling laws for the planets}

Let us now turn to our primary interest: the planets. The first hint that planetary dynamos scale very differently from the numerical dynamos comes from Fig. 3. For the Earth we have $E \sim 10^{-15}$ and $\mathrm{Ra}_{Q} \sim 10^{-12}$, giving $\mathrm{Ra}_{Q} / E \sim 10^{3}$, which lies completely outside of the dipolar regime indicated in Fig. 3. Also $\delta / R_{C} \sim \mathrm{Ek}^{1 / 3}$ requires $\delta \sim 30 \mathrm{~m}$, which is much too small. Of course, this is because the numerical dynamics are strongly influenced by the viscous forces, which set the scale for $\delta$, whereas the viscous stresses play no significant role in planetary cores. So perhaps the key question for the planets is: what dictates the scale $\delta$ ? In this section we shall focus particularly on the Earth, Jupiter and Saturn, partly because these all have dipolar fields, but also because we have plausible estimates of the rate of working of the buoyancy force, $\mathrm{P}$, for these three cases. By way of contrast, there are no reliable estimates of the convective heat flux in Mercury, and the fields of the ice giants are distinctly non-dipolar and so likely to be very different in origin from the gas giants and terrestrial planets.

Let us start with the observational evidence. Table 2 shows some of the dynamo properties of Mercury, Earth, Jupiter and

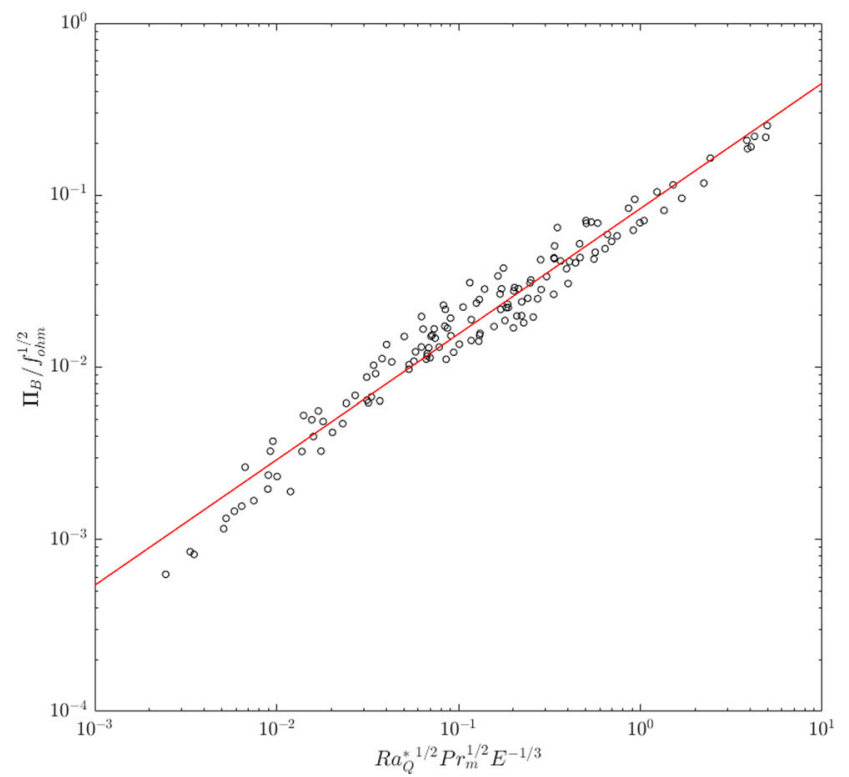

Figure 4. Comparison of scaling laws (41a,b) with the data of Christensen \& Aubert (2006). 
Table 2. Estimates of the Elsasser number, $\Lambda$, and $\bar{B}_{z} / \Omega R_{C} \sqrt{\rho \mu}$ based on the mean axial field in the planetary cores. We use the estimates of $\lambda \sim$ $0.7 \mathrm{~m}^{2} \mathrm{~s}^{-1}, \rho \sim 10^{4} \mathrm{~kg} \mathrm{~m}^{-3}$ for the terrestrial planets, and $\lambda \sim 3 \mathrm{~m}^{2} \mathrm{~s}^{-1}$, $\rho \sim 10^{3} \mathrm{~kg} \mathrm{~m}^{-3}$ for the gas giants. Included for comparison is a fully convective, low-mass, M-dwarf star, which has an 800 Gauss dipole field of the $\alpha^{2}$ type.

\begin{tabular}{lcccc}
\hline $\begin{array}{l}\text { Planet } \\
\text { (or star) }\end{array}$ & $\begin{array}{c}\text { Rotation period } \\
\text { (days) }\end{array}$ & $\begin{array}{c}\text { Elsasser number } \\
\Lambda=\sigma \bar{B}_{z}^{2} / \rho \Omega\end{array}$ & $\frac{\bar{B}_{z} / \sqrt{\rho \mu}}{\Omega R_{C}}$ & $\frac{v_{P}^{2}}{\Omega \lambda}$ \\
\hline Mercury & 58.6 & $2 \times 10^{-4}$ & $5.5 \times 10^{-6}$ & - \\
Earth & 1 & 0.2 & $13 \times 10^{-6}$ & 2.9 \\
Jupiter & 0.413 & 5 & $5.2 \times 10^{-6}$ & 66 \\
Saturn & 0.440 & 0.2 & $2.3 \times 10^{-6}$ & 23 \\
V374 Pegasi & 0.44 & $\sim 10^{4}$ & $17 \times 10^{-6}$ & - \\
\hline
\end{tabular}

Saturn. In order to provide a comparison, we also show data for a fully convective, low-mass, M-dwarf star, V374 Pegasi, which is thought to have an 800 Gauss dipole field of the $\alpha^{2}$ type (Donati et al. 2006). Estimates of the magnetic diffusivities for the planets are constantly changing, but here we take the nominal values of $\lambda \sim 3 \mathrm{~m}^{2} \mathrm{~s}^{-1}, \rho \sim 10^{3} \mathrm{~kg} \mathrm{~m}^{-3}$ for the gas giants (French et al. 2012), and $\lambda \sim 0.7 \mathrm{~m}^{2} \mathrm{~s}^{-1}, \rho \sim 10^{4} \mathrm{~kg} \mathrm{~m}^{-3}$ for terrestrial planets. The mean axial field strengths in the cores, $\bar{B}_{z}$, are obtained from the observed dipole moments using (1), and we use the estimates $P \approx 1.2 \times 10^{-10} \mathrm{~m}^{2} \mathrm{~s}^{-3}, P \approx 0.43 \times 10^{-10} \mathrm{~m}^{2} \mathrm{~s}^{-3}$ and $P \approx 5.1 \times 10^{-13} \mathrm{~m}^{2} \mathrm{~s}^{-3}$ for Jupiter, Saturn and the Earth, respectively, as discussed in Section 5.1.

It is striking that the Elsasser number varies considerably across the various dynamos shown in Table 2 , consistent with the discussion in Section 5.1. By way of contrast, $\left(\bar{B}_{z} / \sqrt{\rho \mu}\right) / \Omega R_{C}$ is relatively uniform across these dynamos, an observation that clearly needs some sort of explanation and holds out hope that universal dynamo scaling laws may indeed exist. Note also that $v_{P}^{2} / \Omega \lambda \geq 1$ for the Earth, and $v_{P}^{2} / \Omega \lambda \gg 1$ for Jupiter and Saturn, which shall turn out to be of some importance in the discussion below.

Perhaps the key question now is whether or not the observational data is consistent with our prediction,

$v_{P}^{3} \sim \frac{v_{a}^{2} \lambda}{\delta} \sim \Omega \delta u^{2}$,

where $v_{a} \sim B_{r m s} / \sqrt{\rho \mu}$. It seems likely that the rms field, $B_{r m s}$, is substantially larger than the mean axial field, $\bar{B}_{z}$, in the planetary cores, and indeed measurements of torsional oscillations in the Earth's core suggest $B_{r m s} \sim 30$ Gauss (Roberts \& King 2013), which is an order of magnitude larger than $\bar{B}_{z}=3.7$ Gauss. Consequently, we shall make the crude estimate $B_{r m s}=10 \bar{B}_{z}$ in what follows.

The primary unknown in (45) is $\delta$, the smallest of the two transverse length-scales associated with the columnar structures lying outside the tangent cylinder. So we now consider two different universal scaling laws that have been suggested for planetary dynamos, each of which implies a different estimate of $\delta$. We shall also tentatively propose an alternative universal scaling law based on the energy estimate (37). To distinguish between these various options we shall insist that $\operatorname{Ro}_{\delta} \sim\left(u / v_{P}\right)^{3} \leq 1$, which is a prerequisite for a dipolar field. Moreover, since (45) yields

$\frac{v_{a}^{2}}{u^{2}} \sim \frac{v_{P}^{2}}{\Omega \lambda} \operatorname{Ro}_{\delta}^{-4 / 3}$,

and we observe that $v_{P}^{2} / \Omega \lambda>1$, the requirement that $\mathrm{Ro}_{\delta} \leq 1$ automatically ensures that $v_{a}^{2} / u^{2}>1$, so that the magnetic energy dominates over the kinetic energy. Note also that $\operatorname{Ro}_{\delta} \leq 1$ combined with (45) rules out the possibility of equipartition, $v_{a}^{2} \sim u^{2}$, which is a common assumption in stellar dynamo modelling.
Table 3. Comparison of observed and predicted values of $\Pi_{B}=$ $B_{r m s} / \Omega R_{C} \sqrt{\rho \mu}$. We use the estimates of $\lambda \sim 0.7 \mathrm{~m}^{2} \mathrm{~s}^{-1}, \rho \sim 10^{4} \mathrm{~kg} \mathrm{~m}^{-3}$ for the Earth, and $\lambda \sim 3 \mathrm{~m}^{2} \mathrm{~s}^{-1}, \rho \sim 10^{3} \mathrm{~kg} \mathrm{~m}^{-3}$ for the gas giants.

\begin{tabular}{lccc}
\hline Planet & $\begin{array}{c}\Pi_{B}=\frac{B_{r m s} / \sqrt{\rho \mu}}{\Omega R_{C}} \\
\text { Observed }\end{array}$ & $\begin{array}{c}\frac{B_{r m s} / \sqrt{\rho \mu}}{\Omega R_{C}} \sim \frac{v_{P}}{\Omega R_{C}} \\
\text { Prediction }(50)\end{array}$ & $\begin{array}{c}\text { Predicted convective } \\
\sqrt{\Omega \lambda} \\
\text { velocity, } u\left(\mathrm{~m} \mathrm{~s}^{-1}\right)\end{array}$ \\
\hline Mercury & $5.5 \times 10^{-5}$ & - & - \\
Earth & $13 \times 10^{-5}$ & $8 \times 10^{-5}$ & 0.01 \\
Jupiter & $5.2 \times 10^{-5}$ & $15 \times 10^{-5}$ & 0.19 \\
Saturn & $2.3 \times 10^{-5}$ & $11 \times 10^{-5}$ & 0.11 \\
\hline
\end{tabular}

Consider first the traditional assumption of $\Lambda=\sigma B_{r m s}^{2} / \rho \Omega \sim 1$. When combined with (45) this yields $u \delta / \lambda \sim 1$ and

$\operatorname{Ro}_{\delta} \sim\left(\frac{v_{P}^{2}}{\Omega \lambda}\right)^{3}>1, \quad \frac{v_{a}^{2}}{u^{2}} \sim\left(\frac{v_{P}^{2}}{\Omega \lambda}\right)^{-3}<1$.

For Jupiter and Saturn this is well into the multipolar regime, and so clearly $\Lambda \sim 1$ is incompatible with (45). Next consider the proposal of Christensen et al. (2009), which is the hypothesis that $v_{a}$ is independent of rotation rate and controlled only by the buoyancy flux: $v_{a} \sim v_{P}$. When combined with (45) this yields $v_{a} \delta / \lambda \sim 1$ and

$\operatorname{Ro}_{\delta} \sim\left(\frac{v_{P}^{2}}{\Omega \lambda}\right)^{3 / 2}>1, \quad \frac{v_{a}^{2}}{u^{2}} \sim\left(\frac{v_{P}^{2}}{\Omega \lambda}\right)^{-1}<1$.

This also lies in the multipolar regime for Jupiter and Saturn. We conclude that, given the observation that $v_{P}^{2} / \Omega \lambda \gg 1$ for the gas giants, both of these scaling proposals are inconsistent with (45).

Cleary we need an alternative criterion for setting the scale $\delta$. Let us, for the moment, retain the hope that a universal scaling law exists and consider the hypothesis that, for a given $\mathrm{P}$, the dynamo minimises its net energy (or equivalently its magnetic energy) while remaining in the dipolar regime. From (37) we see that this requires $u / \Omega \delta \sim 1$, and combined with (45) this yields

$u \sim v_{P}, \quad \frac{v_{a}}{v_{P}} \sim \frac{v_{P}}{\sqrt{\Omega \lambda}}$.

Eq. (40) tells us that this represents the minimum magnetic field strength and minimum length scale, $\delta$, consistent with (45) and with $\operatorname{Ro}_{\delta} \leq 1$. It also ensures the magnetic energy is greater than the kinetic energy. The rather tentative idea behind this hypothesis is that, in the absence of other constraints, a kinematic dynamo growing from a state of weak or zero field will tend to saturate at the minimum field strength consistent with a specified value of the buoyancy flux.

In Table 3 we compare prediction (49) with the observational data for the Earth, Jupiter and Saturn, taking the pre-factors in (45) equal to unity. It is customary to use the dimensionless group

$\Pi_{B}=\frac{B_{r m s} / \sqrt{\rho \mu}}{\Omega R_{C}}=\frac{v_{a}}{\Omega R_{C}}$

when comparing theory or computation with observation for planetary dynamos, and so we shall conform to this convention. In terms of $\Pi_{B}$, (49) becomes

$\frac{B_{r m s} / \sqrt{\rho \mu}}{\Omega R_{C}} \sim \frac{v_{P}}{\Omega R_{C}} \frac{v_{P}}{\sqrt{\Omega \lambda}}$.

Given the uncertainties in the values of the magnetic diffusivities, especially for gas giants, and in the estimates of the convective heat flux, the comparison in Table 3 seems not unreasonable. In particular, note that the observed relative uniformity of $\Pi_{B}$ across 
the planets is reflected in prediction (50). Of course, little credence should be given to the precise numerical values of the predictions in the table, as the pre-factors in (49) may differ significantly from unity. Perhaps the most we should conclude from Table 3 is that the hypothesis of a minimum magnetic energy in the saturated state (for a given $\mathrm{P}$ ) is not inconsistent with (45) and with the observations.

Of course, demanding a minimum in magnetic energy is only one of many possible ways in which the scale $\delta$ might, in practice, be chosen. Indeed, the way that $\delta$ is set may vary from planet to planet, being different, say, for the gas giants and terrestrial planets. For example, in the Earth presumably $\delta$ is also the width of the plumes which meander slowly from the solid inner core to the mantle, and this in turn may be set by the thermal boundary-layer thickness and/or solidification processes at the inner core where the plumes form. So, given the scaling laws (40), and the requirement that $\mathrm{Ro}_{\delta} \leq 1$, perhaps the most that we can reliably conclude is that the predictions of $v_{a}$ and $u$ in Table 3 represent an approximate lower bound on $v_{a}$ and upper bound on $u$, and that the case for universality remains uncertain.

\section{POSSIBLE IMPLICATIONS FOR MERCURY AND FULLY CONVECTIVE STARS}

We close with some speculative comments about Mercury and possible links to dynamos in fully convective stars. We do not know the convective heat flux in Mercury, nor the ratio of the compositional to thermal buoyancy in its core. However, we are free to explore the tentative assumption that Mercury's dynamo operates in the same regime as that of the Earth and the gas giants, governed by (40). If this is indeed the case we can infer from (40) an approximate upper bound on $P$ from the measured value of $\Pi_{B}$. It is $P \approx 2 \times 10^{-17} \mathrm{~m}^{2} \mathrm{~s}^{-3}$, with corresponding estimates of the modified Rayleigh number and convective velocity being $\mathrm{Ra}_{Q} \sim 3 \times 10^{-11}$ and $u \sim 0.3 \mathrm{~mm} \mathrm{~s}^{-1}$. This value of $\mathrm{P}$ is surprisingly low, being a factor of $10^{4}$ lower than that of the Earth and also considerably lower than the estimate used by Christensen (2006) for dynamo simulations based on the thermal history model of Hauck et al. (2004). It is also, perhaps, only an order of magnitude larger than the probable critical value of $\operatorname{Ra}_{Q}$ at which convection shuts down, which is suggestive of extremely weak convection. It should be noted, however, that some dynamo models (e.g. Christensen 2006) suggest that the magnetic field deep within Mercury's convective core is substantially larger than the observable field at the coremantle-boundary, because the outer stratified regions of the core filter out the non-axisymmetric components. If true, the effective value of $\Pi_{B}$ could be much larger, yielding a more supercritical value of $\mathrm{Ra}_{Q}$. In any event, in the absence of compositional buoyancy, $\mathrm{P}$ is related to the convective heat flux by $P=\left(g \beta / \rho c_{p}\right) \dot{q}$, and so the speculative estimate of $\mathrm{P}$ above places potential constraints on the acceptable levels of convective heat flux in thermal evolution models of Mercury. Conversely, independent estimates of this heat flux would help determine whether or not Mercury's dynamo is indeed operating in the same regime as that of the Earth, and so governed by (40).

Turning to stellar dynamos, it is intriguing that the value of $\left(\bar{B}_{z} / \sqrt{\rho \mu}\right) / \Omega R_{C}$ for V374 Pegasi in Table 2 is reasonably consistent with that of the planets. This raises the possibility, already explored in Christensen et al. (2009), that a single scaling law may extend all the way from the planets to fully convective stars with $\alpha^{2}$ dynamos. (Christensen et al. for example, tentatively propose $v_{a} \sim v_{P}$ across the full range.) However, perhaps some caution is required here, as such stars are not as rotationally constrained as the planets, having much higher convective velocities and hence larger Rossby numbers. The point is that the convective heat flux in such stars is very high, leading to large values of $v_{P}$, and hence of $u$ and $v_{a}$. If the Rossby number at the small scales exceeds unity, which seems likely in the light of the large Elsasser number in Table 2, the flow will cease to be dominated by columnar structures aligned with the rotation axis, and scaling analyses of the type presented here fail because buoyancy is then balanced by inertia. For example, luminosity measurements of V374 Pegasi give $\dot{q} \approx 4 \times 10^{6} \mathrm{~W} \mathrm{~m}^{-2}$ and hence $v_{P} \sim 30 \mathrm{~m} \mathrm{~s}^{-1}$, which is three orders of magnitude higher than typical estimates of $v_{P}$ for the planets. If the resulting smallscale Rossby number exceeds unity, then scaling (49) must fail, and indeed (49) predicts a magnetic field strength greatly in excess of the observed field of around 800 Gauss.

A common alternative suggestion for fully convective stars, based on the assumption that the small-scale Rossby number is moderateto-large (and hence the Coriolis force weak), is the triple force balance

$\nabla \times(\rho \mathbf{u} \cdot \nabla \mathbf{u}) \sim \nabla \times\left(\rho^{\prime} \mathbf{g}\right) \sim \nabla \times(\mathbf{B} \cdot \nabla \mathbf{B} / \mu)$.

Assuming a single length-scale for all gradients, $\ell$, this yields

$\frac{u^{2}}{\ell^{2}} \sim \frac{P}{u \ell} \sim \frac{B^{2}}{\rho \mu \ell^{2}}$,

and hence the equipartition scaling $u \sim v_{a} \sim \hat{v}_{P}$, where $\hat{v}_{P}=$ $(P \ell)^{1 / 3}$. If we further assume $\ell=R_{C}$, this takes us back to $\Pi_{B} \sim \Pi_{P}{ }^{1 / 3}$, as suggested in Christensen et al. (2009). However, the observed field strength for V374 Pegasi is around 800 Gauss, and so a mean density of $15 \times 10^{3} \mathrm{~kg} \mathrm{~m}^{-3}$ results in $v_{a} \sim 0.6 \mathrm{~m} \mathrm{~s}^{-1}$. Evidently, for this particular star, the equipartition argument combined with $\ell=R_{C}$ significantly overestimates the rms field strength. Reducing the estimate of the characteristic scale $\ell$ could, in principle, resolve this discrepancy, although a reduction in $\ell$ by a factor of around $10^{5}$ would be required to bring the observed and predicted field strengths into line. So, one way or another, it seems that the scaling laws for dynamos in fully convective stars remains elusive.

\section{CONCLUSIONS}

We have shown that, given the balance between energy production and Joule dissipation, and between the curl of the buoyancy and Coriolis forces, planetary dynamos should satisfy the scaling relationship $v_{P}^{3} \sim v_{a}^{2} \lambda / \delta \sim \Omega \delta u^{2}$. To arrive at this scaling relationship we have estimated the Joule dissipation using the helical-wave dynamo cartoon of Davidson \& Ranjan (2015), but in fact this estimate really only requires $\mathbf{j} \sim \sigma \mathbf{v} \times\left\langle\mathbf{B}_{\perp}\right\rangle$, which is likely to hold in a wide range of dynamos, including those driven by Ekman pumping. So our scaling analysis should be valid across a broad range of dipolar, $\alpha^{2}$ dynamos. Certainly it is consistent with the evidence of the numerical dynamos, where $\delta$ is set by the viscous forces, yielding predictions consistent with published empirical scaling laws and also with the observed transition from dipolar to multipolar dynamos. A direct comparison with the observational evidence for the planets is hampered by the fact that we do not know how the scale $\delta$ is set in the planets. Nevertheless, we have shown that the traditional assumption of $\Lambda \sim 1$ is inconsistent with the observation that the gas-giant dynamos are dipolar, as is the suggestion of Christensen et al. (2009) that $v_{a}$ is independent of rotation rate and controlled only by the buoyancy flux: $v_{a} \sim v_{P}$. On the other hand, 
we have shown that the hypothesis that $\delta$ is set by $u / \Omega \delta \sim 1$ is consistent with the observations, and in particular it is consistent with the observation that $\Pi_{B}$ is relatively uniform across the planets, of order $10^{-4}$. The criterion $u / \Omega \delta \sim 1$ corresponds to the assertion that dipolar dynamos saturate at the lowest permissible magnetic energy consistent with a given buoyancy flux. More generally, we have established that: (i) scaling laws deduced from the numerical dynamos cannot, in general, be extended to the planets (see Fig. 3); and (ii) there exists an approximate upper bound for $u$ and a lower bound for $v_{a}$ for the dynamos of the Earth, Jupiter and Saturn (eq. 40).

\section{ACKNOWLEDGEMENTS}

The author thanks Andy Jackson, Uli Christensen, Oli Bardsley and Avishek Ranjan for their helpful comments.

\section{REFER E N CES}

Aubert, J., Finley, C.C. \& Fournier, A., 2013. Bottom-up control of geomagnetic secular variation by the Earth's inner core, Nature, 502, 219-223.

Bin Baqui, Y. \& Davidson, P.A., 2015. A phenomenological theory of rapidly-rotating turbulence, Phys. Fluids, 27(2), doi:10.1063/1.4907671.

Christensen, U.R., 2006. A deep dynamo generating Mercury's magnetic field, Nature, 444, 1056-1058.

Christensen, U.R., 2010. Dynamo scaling laws and application to the planets, Space Sci. Rev., 152, 565-590.

Christensen, U.R., 2011. Geodynamo models: tools for understanding properties of Earth's magnetic field, Phys. Earth planet. Inter., 187, 157-169.

Christensen, U.R. \& Aubert, J., 2006. Scaling properties of convectiondriven dynamos in rotating spherical shells and applications to planetary magnetic fields, Geophys. J. Int., 166, 97-114.

Christensen, U.R. \& Wicht, J., 2007. Numerical dynamo simulations, in Treatise on Geophysics, ed. Olson, P., Elsevier.

Christensen, U.R., Holzwarth, V. \& Reiners, A., 2009. Energy flux determines magnetic field strength of planets and stars, Nature, 457, 167-169.

Davidson, P.A., 2013. Turbulence in Rotating, Stratified and Electrically Conducting Fluids, Cambridge Univ. Press.

Davidson, P.A., 2014. The dynamics and scaling laws of planetary dynamos driven by inertial waves, Geophys. J. Int., 198 (3), 1832-1847.
Davidson, P.A. \& Ranjan, A., 2015. Planetary dynamos driven by helical waves: Part 2, Geophys. J. Int., 202, 1646-1662.

Donati, J.-F., Forveille, T., Cameron, A.C., Barnes, J.R., Delfosse, X., Jardine, M.M. \& Valenti, J.A., 2006. The large-scale axisymmetric magnetic topology of a very-low-mass fully-convective star, Science, 311, 633-635.

French, M., Becker, A., Lorenzen, W., Nettelmann, N., Bethkenhagen, M., Redmer, R. \& Wicht, J., 2012. Ab initio simulations for material properties along the Jupiter adiabat, Astrophys. J. Suppl. Ser., 202(5), doi:10.1088/0067-0049/202/1/5.

Glatzmaier, G.A. \& Roberts, P.H., 1995. A three-dimensional convective dynamo solution with rotating and finitely conducting inner core and mantle, Phys. Earth planet. Inter., 91, 63-75.

Hauck, S.A., Dombarda, A.J., Phillipsc, R.J. \& Solomona, R.J., 2004. Internal and tectonic evolution of Mercury, Earth planet. Sci. Lett., 222, 713-728.

King, E.M. \& Buffett, B.A., 2013. Flow speeds and length scales in geodynamo models: the role of viscosity, Earth planet. Sci. Lett., 371-372, $156-162$.

Olson, P., Christensen, U.R. \& Glatzmaier, G.A., 1999. Numerical modelling of the geodynamo: mechanisms of field generation and equilibration, $J$. geophys. Res., 104(B5), 10 383-10 404.

Oruba, L. \& Dormy, E., 2014. Predictive scaling laws for spherical rotating dynamos, Geophys. J. Int., 198, 828-847.

Read, P.L. et al., 2015. Global energy budgets and 'Trenberth diagrams' for the climates of terrestrial and gas giant planets, Q. J. R. Meteorol. Soc., 142, 703-720.

Roberts, P.H. \& King, E.M., 2013. On the genesis of the Earth's magnetism, Rep. Prog. Phys., 76, doi:10.1088/0034-4885/76/9/096801.

Sakuraba, A. \& Roberts, P.H., 2009. Generation of a strong magnetic field using uniform heat flux at the surface of the core, Nat. Geosci., 2, 802805 .

Schrinner, M., Petitdemange, L. \& Dormy, E., 2012. Dipole collapse and dynamo waves in global direct numerical simulations, Astrophys. J., 752(2), 121, doi:10.1088/0004-637X/752/2/121.

Stanley, S. \& Glatzmaier, G.A., 2010. Dynamo models for planets other than the Earth, Space Sci. Rev., 152, 617-649.

Stelzer, Z. \& Jackson, A., 2013. Extracting scaling laws from numerical dynamo models, Geophys. J. Int., 193(3), 1265-1276.

Yadav, R.K., Gastine, T. \& Christensen, U.R., 2012. Scaling laws in spherical shell dynamos with free-slip boundaries, Icarus, 225, 185-193. 